\title{
Hepatitis B immunisation among invasive cardiologists: poor compliance with United Kingdom guidelines
}

\author{
B D Prendergast, N P Andrews, A Thomas, L Davies, M McCabe, W J Penny
}

\begin{abstract}
Objectives-To assess the compliance of invasive cardiologists in the United Kingdom with recently accepted national guidelines on the protection of health care workers and patients from hepatitis $B$. To determine levels of awareness of the infectivity and prevalence of the virus and current attitudes towards screening of patients before cardiac catheterisation and surgery.

Design-Anonymous postal survey by questionnaire from the University Hospital of Wales, Cardiff. The questionnaire established the respondent's position, knowledge of hepatitis $B$, current immunological state, and policy towards the routine screening of patients for hepatitis B carriage.
\end{abstract}

Participants-All British cardiologists of consultant or senior registrar grade involved in invasive procedures.

Results-The response rate was $78 \%$ (211/271). $20 \%$ of respondents had never been vaccinated against hepatitis $B$ and about a third of those vaccinated had not complied correctly with the recommended immunisation regimen. There was little uniformity in practices for screening patients for hepatitis $B$ carriage before invasive procedures, and the level of knowledge concerning the prevalence of hepatitis $B$ and the risks of inoculation was poor.

Conclusions-Invasive cardiologists are at high risk of inoculation with hepatitis B. Nationally agreed guidelines are designed to protect both medical staff and patients against the risk of infection but currently they are ill heeded.

(Br Heart f 1995;74:685-688)

Wales, Cardiff

B D Prendergast

N P Andrews

A Thomas

L Davies

W J Penny

Morriston Hospital,

Swansea

M McCabe

Correspondence to:

Dr W J Penny, Consultant

Cardiologist, University of

Accepted for publication

12 June 1995 borne infection, most notably hepatitis human immunodeficiency virus (HIV), are well recognised. While debate persists regarding the management of those occupationally exposed to HIV, there are firm guidelines for the protection of health care workers and patients from hepatitis $B .^{1}$

Currently about 200 million people worldwide carry the hepatitis B virus. ${ }^{2}$ In the United Kingdom 1 in $1500(0.07 \%)$ new blood donors carry the virus and recent antenatal clinic data suggest an estimated prevalence of $1 \%$ in inner city areas. About $10 \%$ of carriers are highly infective because they are hepatitis B e antigen positive. ${ }^{1}$ The potential consequences of hepatitis $B$ infection are serious and well known. Moreover, the risk of passage to others is high, either by sexual or bloodborne means: the rate of seroconversion after injury with a contaminated needle stick from a patient with hepatitis $B$ ranges from $5 \%^{3}$ to $30 \%{ }^{4}$ when blood is e antigen positive.

In the United Kingdom the number of overt cases of hepatitis B is low and there has been a considerable decrease in recent years. Notifications to the Public Health Laboratory Service fell from a peak of almost 2000 in 1984 to about 500 in $1990 . .^{5}$ Between 1985 and 1988, 139 cases of acute hepatitis B were reported among health service staff-this was $3.1 \%$ of the total notified. Compared with previous years this was a fall, probably owing to vaccination. ${ }^{6}$ Furthermore, between 1975 and 1990 twelve outbreaks of hepatitis B, totalling 95 infections, were ascribed to transmission of virus from hospital staff to patients. ${ }^{7}$ Inevitably, these figures are an underestimate because of failure of notification, the prolonged incubation period of the virus, and inapparent cases of subclinical infection.

In 1987 an inexpensive recombinant yeast derived hepatitis B vaccine (Engerix B, SmithKline Beecham) became available. This replaced the previous plasma derived preparation which had been associated with the fear of transmission of other infective agents. Comprehensive guidelines for its use were established and these were updated in $1993 .^{1}$ In particular, it is recommended that "all health care workers who perform exposure prone procedures... or who are at risk of acquiring hepatitis B occupationally from blood-stained sharp instruments . . . should be immunised ... unless immunity as a result of natural infection or previous immunisation has been documented". Despite such clear 
instructions the available data suggest that hepatitis $B$ vaccine uptake is poor.

Therefore, the aim of this study was to assess the compliance of invasive cardiologists with these guidelines and to determine their awareness of the infectivity and prevalence of the virus.

\section{Methods}

The survey was approved by the British Cardiac Society. All United Kingdom cardiologists of consultant and senior registrar grade involved in invasive procedures were contacted. Questionnaires were posted in July 1993, followed five months later by one reminder to those who had not responded. All replies were anonymous.

The questionnaire (figure) was designed to address three main issues:
Awareness of the infectivity of hepatitis B virus and its prevalence in the community

- The level of uptake of hepatitis B vaccine, including completion of the vaccination regimen, and reasons for non-compliance

- The prevalence of hepatitis B screening policies and their utilisation

Statistical analysis was undertaken using Fisher's exact test; a probability $(\mathrm{P})$ value $<0.05$ was regarded as significant.

\section{Results}

We received a reply from $211(77.9 \%)$ of the 271 cardiologists $(175(83 \%)$ consultants and $36(17 \%)$ senior registrars) surveyed, a favourable response rate compared with previous mail surveys among hospital medical personnel..$^{8}$

The general level of knowledge concerning

Please answer questions $1-3$ by encircling the appropriate answer or the answer you consider to be correct

\section{Current clinical grade:}

Registrar / Senior Registrar / Consultant

\section{Concerning Hepatitis B virus}

(a) The current estimate of hepatitis B carrier state prevalence in the UK is in the order of 1 in $500 \quad 1$ in $5,000 \quad 1$ in 50,000

(b) The risk of acquiring hepatitis $B$ infection following an inoculation accident involving surface antigen (HBsAg) positive ('e' antigen ( $\mathrm{HBeAg}$ ) negative) blood is $0.2 \% \quad 2 \% \quad 20 \%$

(c) The risk when the inoculation accident involves $\mathrm{HBeAg}$ positive blood is $0.2 \% \quad 2 \% \quad 20 \%$

\section{Concerning hepatitis B immunisation}

(a) How many vaccine injections have you received?

(b) Have your antibody levels been checked? Yes / No

(c) If you have not completed the immunisation schedule, what is the reason for this?
i) Risk too low to warrant immunisation
iv) Never offered immunisation
ii) Already immune
v) Intend to, but yet to find time
iii) Other (please state)
vi) Concerned about safety of vaccine

Please answer question 4 by marking the analogue scale with a vertical line at the point which you feel reflects the practice in your unit

\section{Concerning routine screening of patients for hepatitis B infection.}

(a) What proportion of cardiac catheterisation patients are screened?

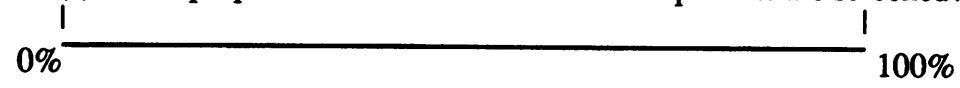

(b) Are the hepatitis B results known before catheterisation?

never

(c) Are patients who undergo cardiac surgery screened immediately before surgery?

never $\quad$ always


the prevalence of hepatitis $B$ carriage and the risks of inoculation was poor. Of 188 respondents only $65(34.6 \%)$ were aware of the true prevalence and $27(14.4 \%)$ were incorrect by a factor of 100 . The risk of acquiring hepatitis $B$ after accidental inoculation with e antigen positive blood was estimated correctly by $108 / 181$ $(59 \cdot 7 \%)$ respondents and $18(9.9 \%)$ were incorrect by a factor of 100 .

Collectively, $42(19.9 \%)$ of the respondents had never been vaccinated against hepatitis $B$ (consultants $v$ senior registrars, NS). Of those vaccinated, only $111 / 169(65 \cdot 6 \%)$ had completed a course of three vaccinations and $54 / 169(32 \cdot 0 \%)$ had not had antibody levels checked subsequently to ensure immunity had been achieved. Eighty two respondents provided a reason for failing to comply with the immunisation regimen: the most commonly cited explanation was lack of time (54 respondents), 11 had never been offered vaccination by their occupational health department, and one had received confusing advice regarding immunisation.

There is wide variation in the practice of screening patients for hepatitis B carriage before cardiac catheterisation and surgery: $67 / 211(31 \cdot 8 \%)$ of cardiologists never screen before cardiac catheterisation and 52/211 $(24 \cdot 6 \%)$ screen rarely ( $<20 \%$ of patients), $31 / 211(14 \cdot 7 \%)$ screen virtually all $(>80 \%)$ patients; and only $38 / 211(18.0 \%)$ screen routinely. Two respondents were unaware of the screening practice on their own unit. The results of screening are never, or rarely $(<20 \%)$, known before catheterisation by $117 / 211(55.5 \%)$ of cardiologists and only available routinely to $19 / 211(9 \cdot 0 \%)$. Only $58 / 211(27 \cdot 5 \%)$ screen patients who proceed to cardiac surgery and $74 / 211(35.0 \%)$ never, or rarely, screen in this situation.

\section{Discussion}

This survey highlights poor compliance with national guidelines on occupational hepatitis $B$ infection among a medical group regularly exposed to the risk of accidental inoculation. Although a vaccination rate of $80.1 \%$ was achieved, about a third of vaccinated cardiologists had not completed the course of injections or had their immunity confirmed. There was little uniformity in the practice of screening patients before invasive cardiac procedures. It seems that many units selectively screen those perceived to represent a high risk, usually because of their country of origin (particularly the Middle East, Africa, and the Mediterranean) but the results of screening are very rarely known before catheterisation. Several centres screen patients before heart transplantation but not before routine cardiac surgery.

Previous studies among medical staff also showed poor vaccine uptake: in the United States an estimated $24 \%$ of primary physicians have been vaccinated ${ }^{9}$ and, in the United Kingdom, Kinnersley ${ }^{10}$ reported a $48 \%$ vaccination rate in general practitioners. Among hospital-based medical staff, where perceived risks are higher, these figures are little improved. In 1990 a survey by interview of 100 medical staff in a Manchester teaching hospital found a vaccination rate of $49 \%,{ }^{11}$ of whom about a third had not had immunity confirmed with subsequent serological analysis. The most commonly cited reasons for poor compliance were apathy and difficulty in obtaining the vaccine. Among paediatricians a hepatitis $B$ immunisation uptake of $71 \%$ has been reported, ${ }^{12}$ and several surveys, similar to our own, among surgeons showed rates of vaccine uptake between $64 \%$ and $90 \% . .^{13-15}$

The recommendations of the National Advisory Group on hepatitis published in August 1993 stipulate that all doctors whose work involves exposure prone procedures should be immunised against hepatitis B unless they are known to be naturally immune. ${ }^{1}$ The basic immunisation regimen consists of three intramuscular doses of vaccine given at 0,1 , and 6 months. About $10 \%$ (higher in those $>40$ years) do not respond to this primary course and thus immune status should be checked 2-4 months later. A hepatitis B surface antigen antibody titre of $>100$ $\mathrm{mIU} / \mathrm{ml}$ confers protective immunity and is regarded an adequate response. Levels between 10-100 $\mathrm{mIU} / \mathrm{ml}$ may not necessarily confer long-lasting protection, and a booster dose may be required. It is hoped that newer vaccines currently being developed will increase the response rate.

A repeat course of immunisation is recommended for those who do not respond (hepatitis B surface antigen antibody titre $<10$ $\mathrm{mIU} / \mathrm{ml}$ at $2-4$ months). In some cases this may be due to chronic carriage of the hepatitis $B$ virus and those who fail a second time should be referred for specialist advice and, after appropriate counselling and consent, their hepatitis B carrier status determined. Those found in this way to be e antigen positive should curtail exposure prone procedures for the protection of patients and other staff. In some, treatment with interferon may prove appropriate. This has been reported to reverse carriage status in up to $40 \%$ of cases. ${ }^{16}$ There are no documented outbreaks involving transmission from hepatitis B surface antigen positive health workers who are e antigen negative; in these circumstances such an individual need not be barred from any area of work.

The duration of immunity after vaccination is not known. At present it is suggested that those who continue to be exposed to the risk of infection should receive a booster dose 3-5 years after initial immunisation, unless this has already been required after possible exposure to the virus.

Various schemes have been proposed to increase vaccination rates among those at risk. These have included screening at the time of pre-employment medicals or before starting training in invasive cardiology. Periodic immunisation visits to cardiac catheter laboratories with appropriate publicity have also been advocated. Finally it is now recommended policy that medical students should be routinely immunised against hepatitis $B$ 
before any clinical component of their course; recent recommendations that all applicants to medical school should be screened, and immunised if appropriate, have far-reaching implications and have provoked controversy. ${ }^{17} 18$

In conclusion, among invasive cardiologists in the United Kingdom there is clear scope for improvement in vaccine uptake, particularly in completion of the immunisation regimen. Screening of patients, as recommended, should also be performed. Careful practice to avoid needle stick injury should continue and immunisation should also be offered to nursing and other ancillary staff caring for patients undergoing invasive cardiac procedures.

We thank Dr J Heptonstall, Consultant Medical Microbiologist, Public Health Laboratory Service Communicable Disease Surveillance Centre, London for invaluable advice before the preparation of this report. We also thank all those who completed and returned the questionnaire.

1 Recommendations of the Advisory Group on Hepatitis. Protecting health care workers and patients from hepatitis B. HMSO, London 1993.

2 Zuckermann A. Who should be immunised against hepatitis B? BMF 1984;289:1243

3 Recommendations of the Expert Advisory Group on AIDS. Guidance for clinical health care workers: protection against infection with HIV/hepatitis B. HMSO, London 1990.

\section{CORRECTIONS}

Failure of "effective" treatment for heart failure to improve normal customary activity. $\mathcal{F} T \mathrm{Walsh}, R$ Andrews, A Evans, A f Cowley (Br Heart $\mathcal{f}$ 1995;74: 373-6).

Exercise ventilation after balloon dilatation of the mitral valve. A P Banning, N P Lewis, $\mathcal{f} S$ Elborn, $R \mathcal{F}$ $C$ Hall (Br Heart $\mathcal{F} 1995 ; 74: 386-9$ ).

We regret that the incorrect volume number was given in the strap lines and at the foot of the abstracts of these papers. The correct volume number is 74

Permanent pacemaker practice at a Scottish district general hospital between 1987 and 1994. $7 \mathrm{G}$ Doherty, F Dawson, F Kerr

We regret that an error appeared in the second sentence of the discussion on page 477 of this article $(\mathrm{Br}$ Heart $\mathcal{F}$ 1995;73:475-8) which should have read, "Our average implantation rate over the 79 months $(130 / \mathrm{mil}-$ lion population/year) compares with the United Kingdom national 1989 value of 148 /million population/year." $"$
4 Centers for Disease Control guidelines for prevention of HIV and hepatitis B viruses to health care and public safety workers. MMWR 1989;38(suppl 56, 4-5):31-3.

5 Department of Health. Immunisation against infectious diseases. London: HMSO, 1992:110-9.

6 Polakoff S. Acute viral hepatitis B, reported to the Public Health Laboratory Service. F Infect 1990;20:163-8.

7 Heptonstall J. Outbreaks of hepatitis B virus infection associated with infected surgical staff. Communicable Disease Report 1991;1(8):R81-5

8 Cartwright A. Professionals as poor responders: variations in and effects of response rates to questionnaires 1961-77. BMF 1978;ii:1419-21.

9 Editorial. Few physicians vaccinated against hepatitis B. Wis Med 7 1989;88:14.

10 Kinnersley $P$. Attitudes of general practitioners towards their vaccination against hepatitis B. BMF 1990;300:238.

11 Burden AD, Whorwell PJ. Poor uptake of hepatitis B immunisation amongst hospital-based health care staff Postgrad Med F 1991;67:256-8.

2 Buss PW, McCabe M, Verrier Jones ER. Attitudes of paediatricians to HIV and hepatitis B virus infection. Arch Dis Child 1991;66:961-5.

13 Berridge DC, Galea MH, Evans DF, Pugh S, Hopkinson BR, Makin GS. Hepatitis B immunisation in vascula surgeons. Br F Surg 1990;77:585-6.

14 Williams JR, Flowerdew ADS. Uptake of immunisation against hepatitis $\mathrm{B}$ among surgeons in Wessex Regional Health Authority. BMF 1990;301:154.

15 Le F, Porteous MJ. Operating practices of and precautions taken by orthopaedic surgeons to avoid infection with taken by orthopaedic surgeons to avoid infection with 301:167-9.

16 Lever AML. Treatment of the chronic hepatitis B virus carrier state. F Infect 1988;16:221-9.

17 Lever AML. Editorial: Hepatitis B and medical studen admission. BMF 1994;308:870-1.

8 Kingman $S$. Hepatitis B status must be known for medical school. BMF 1994;308:876. Cardiac Society will take place at the Scottish Exhibition \& Conference Centre, Glasgow from 7 to 9 May.

The Fourth Annual Molecular Symposium at UCL Medical School on Ischaemic Preconditioning and Adaptation to Ischaemia will take place on 12 December 1995 in London. For further information please contact Jan Wenley, Symposium Administrator, Department of Molecular Pathology, 46 Cleveland Street, London W1P 6DB (tel: +44 171380 9343; fax: +44 1713873310 ). 